\title{
Computando la vida social: \\ el nuevo papel de los métodos de análisis sociológico en Internet
}

\author{
Pablo Navarro \\ Universidad de Oviedo \\ pnavarro@uniovi.es
}

RESUMEN

Las técnicas de investigación social — desde el análisis de contenido a las diversas formas de análisis multivariante- están llamadas a experimentar una transformación radical como consecuencia de la irrupción del fenómeno Internet. De ser instrumentos al servicio de un conocimiento especializado, esas técnicas van a convertirse en mediadoras directas, en tiempo real, de las interacciones sociales en la Red. En Internet, determinadas formas de interacción social pueden autocomputarse en el propio proceso de su desarrollo; gracias a la Red, es posible realizar esta autocomputación en tiempo real y de manera automática. De ahí que buena parte de las técnicas clásicas de la investigación social, cuando se emplean en Internet, pasen a convertirse en técnicas de interacción virtual, generadoras de una nueva dimensión de la socialidad humana. Una socialidad artificial cuyo desarrollo va a tener, probablemente, consecuencias tan profundas como las entrañadas por otros dispositivos de interacción virtual inventados con anterioridad —-por ejemplo, el dinero—.

Palabras clave: Sociología de Internet, Teoría Sociológica, Técnicas de Investigación Social, Socialidad Artificial, Autocomputación Social, Interacción Virtual, Metainformación. 


\section{INTRODUCCIÓN}

Pensé que tendría un impacto asombroso... llegar a analizar la estructura de la sociedad a través de lo que sucede en la Red.

Tim Berners-Lee, inventor de la World Wide Web (citado por Jack D. Stein en netmani@, n.를 25. 53)

El objetivo de este trabajo es apuntar a una transformación decisiva - a una singular metamorfosis- por la cual las técnicas de investigación social parecen destinadas a ser, cada vez más, no sólo instrumentos de análisis de la realidad social, sino también factores directamente constitutivos de ésta. La indicada transformación tendría como causa y como entorno el actual desarrollo de Internet. Merced a este desarrollo, las referidas técnicas, además de producir conocimiento acerca del objeto social, están empezando a configurar ese objeto de forma directa. Veamos cómo.

La teoría sociológica ha venido subrayando el hecho ${ }^{1}$ de que entre el conocimiento sociológico y la realidad social se da una relación reflexiva: esa realidad, que está en el origen del aludido conocimiento, resulta a su vez transformada por éste. Es evidente, en este sentido, que la aplicación heurística de las técnicas de investigación social revierte en el objeto que estudian, pues contribuye indirectamente - a través del conocimiento por ellas generado- a la transformación de ese objeto.

En nuestros días, sin embargo, la relación entre las variadas técnicas empleadas en la investigación sociológica y la realidad social lleva camino de hacerse reflexiva en otro sentido mucho más directo. Esas técnicas, en el entorno de interacción social mediada por computadora que es Internet, están llamadas a jugar un papel hasta ahora inédito: el de constituirse en el instrumento básico capaz de producir y reproducir, dentro de ese peculiar entorno, toda una red dinámica de interacciones sociales implícitas, complejas y operantes en tiempo real.

De este modo, las referidas técnicas no van ya sólo a mantener su tradicional relación reflexiva indirecta — vía el conocimiento sociológico que su uso posibilita - con la realidad social. Ahora, además, se aprestan a entablar con esa realidad una relación reflexiva mucho más directa e íntima, fundada en su capacidad de actuar como auténticos dispositivos mediadores de la dinámica de la interacción social entre los agentes que se expresan y actúan en la Red. Las consecuencias sociogenéticas de esta nueva función que las mencio-

1 Véase E. Lamo de Espinosa (1990). 
nadas técnicas se hallan en trance de asumir no son fáciles de prever, pero serán sin duda de la mayor trascendencia para la evolución de la neomoderna sociedad planetaria que actualmente se halla en proceso de constitución.

\section{2. ¿HACIA UNA SOCIEDAD DE LA METAINFORMACIÓN?}

La sociedad actual —afirman prestigiosos autores - se distingue por ser, cada vez más, una sociedad de la información ${ }^{2}$. Esta caracterización es, sin duda, brillante y sugestiva: parece capturar un ingrediente peculiar de nuestro mundo, que lo diferenciaría de lo que han sido estadios anteriores de la modernidad. Sin embargo, miradas las cosas más de cerca, nos damos cuenta de que esa caracterización requiere ulteriores precisiones. Pues, de cierto, todas las sociedades humanas, y no sólo ésta, han manejado información y se han constituido sobre la base de procesos de comunicación de esa información — todas han sido, en este sentido, «sociedades de la información»-. Lo que resulta peculiar de la presente sociedad, a este respecto, es la forma específica como en ella se produce la información, y el modo como ésta circula, se recombina y se distribuye a través del cuerpo social.

\section{El hecho de la metainformación}

Cuando se enfoca el problema desde esta exigencia de una mayor precisión conceptual, la sociedad presente no se caracteriza tanto por ser una sociedad de la información — todas las sociedades lo son- como por ser una sociedad crecientemente sometida al imperio de lo que podríamos denominar metainformación $n^{3}$. La nuestra es, en efecto, una sociedad que genera y utiliza, en cantidades crecientes, un tipo nuevo y muy específico de información. Este peculiar género de información tiene como objeto, precisamente, la producción de información — de ahí que el nombre de «metainformación» le resulte apropiado-. El nuevo y expansivo universo de la metainformación está constituido por recursos y herramientas informacionales -en esencia, por dispositivos de procesamiento de información en formato digital - destinados justamente a facilitar otras informaciones efectivamente utilizables por los usuarios. Lo que esos recursos y herramientas hacen es tipificar, formatear y controlar estas últimas informaciones, no sólo en su proceso de elaboración, sino también en el de su circulación, recombinación, distribución y uso social.

2 Véase M. Castells (2001).

3 Para una explicación más pormenorizada del concepto de «metainformación», véase P. Navarro (2002). 
Repárese en que lo característico de esta realidad a la que damos el nombre de metainformación no estriba en que sea, sin más, una herramienta de «producción de información por medio de información». Pues, por ejemplo, una conversación cara a cara -en la que no interviene ningún elemento de naturaleza metainformacional- también sería un proceso de «producción de información por medio de información». Lo que resulta peculiar de la sociedad actual, y de la metainformación que en ella prolifera, es algo más específico, que consiste en lo siguiente: en nuestro mundo, y de manera cada vez más acusada, la información efectivamente comunicada, interpretada y en cierto modo «consumida» como tal por los individuos (la que podemos llamar información de comunicación) está siendo crecientemente generada por medio de un tipo de información nuevo, distinto y especializado. Esta segunda clase de información, que cabe denominar información de procesamiento, no es directamente "consumible» por sus usuarios — no encierra significado sustantivo alguno para ellos-, sino que se limita a describir y prescribir aspectos de la «información de comunicación» efectivamente interpretable por éstos.

Es conocida la distinción que la filosofía del lenguaje establece entre «lenguaje objeto» y «metalenguaje». El lenguaje objeto ${ }^{4}$ habla de algún tipo de realidad extralingüística. El metalenguaje se refiere exclusivamente al lenguaje objeto — por eso precisamente es éste su objeto-. La «información de comunicación», efectivamente interpretable y «consumible» por los sujetos que la emplean, sería equivalente al lenguaje objeto. En tanto que la «información de procesamiento», no directamente «consumible» y que se limita a definir aspectos de la correspondiente información de comunicación, cumpliría una función similar a la de un metalenguaje. De ahí que tenga sentido denominar «metainformación» a esta segunda suerte de información ${ }^{5}$.

Así como en el lenguaje verbal no existe ninguna distinción clara, sistemática y permanente entre lenguaje objeto y metalenguaje ${ }^{6}$, en las sociedades anteriores a la nuestra la información de comunicación y la información de procesamiento han operado casi siempre de manera entremezclada. Nuestra cultura tecnológica actual, por el contrario, distingue cada vez más y de modo más sistemático entre esas dos clases de información, y concede un papel crecientemente importante a la segunda. Este papel se ejerce por medio de ciertos

\footnotetext{
4 En el sentido de «lenguaje objeto último», es decir, lenguaje objeto que no es, a su vez, el metalenguaje de otro lenguaje objeto. Un metalenguaje, en efecto, puede ser el lenguaje objeto de otro metalenguaje de nivel superior. Véase J. Ferrater Mora (1979).

5 Conviene no pasar por alto, sin embargo, que existen sustanciales diferencias entre los conceptos de metainformación y metalenguaje. A este respecto, véase P. Navarro (op. cit.).

6 Ésta es una de las características más peculiares del lenguaje verbal, la cual estriba en la capacidad que tiene la conciencia humana de saltar sin esfuerzo - y con una porción tolerable de confusiones— entre distintos niveles lógicos y modales. Véase P. Navarro (1994).
} 
lenguajes artificiales, aplicados a mecanismos de procesamiento de información - generalmente, de tipo digital - igualmente artificiales y cada vez más refinados y potentes. Así, en nuestros días la información de procesamiento, que nuestra sociedad produce con una profusión cada vez mayor, tiende progresivamente a tomar cuerpo mediante artefactos de carácter metainformacional —en vez de generarse en exclusiva, como ocurría antes, a través del uso de instrumentos naturales, como el lenguaje verbal-.

El ejemplo más cercano y conocido de dispositivo metainformacional es, sin duda, el programa informático - por ejemplo, el procesador de texto que me permite redactar estas páginas-. El programa informático es el motor metainformacional que permite configurar, transmitir, distribuir y hacer accesible la información de comunicación digitalmente procesada por los medios de computación modernos ${ }^{7}$.

\section{La metainformación en la Red}

Hasta la difusión masiva de Internet, las tareas metainformacionales que dan forma al moderno procesamiento digital de la información eran realizadas por computadoras aisladas o, como mucho, quedaban restringidas al dominio de redes locales de computadoras. Mas cuando las máquinas que asumían esas tareas pasaron a estar conectadas a la Red de redes, la situación cambió drásticamente. En esas nuevas condiciones, tanto los recursos informacionales de cada computadora - sus datos - como sus recursos metainformacionales - los programas y metadatos ${ }^{8}$ que procesan esos datos- ya no son simplemente aquellos que la máquina controla directamente, sino los de la Red en su conjunto - $\mathrm{O}$, mejor dicho, los de la Red accesible para esa computadora-.

El cambio de escala que experimenta la información en formato digital cuando pasa de su condición inicial, localmente circunscrita, a estar disponible en Internet torna imprescindible y urgente la invención de nuevos mecanismos metainformacionales. La producción de esas herramientas metainformacionales, que tienen ya la Red entera como dominio propio de aplicación, y que cuentan con características cada vez más potentes, complejas y varia-

\footnotetext{
7 Es decir, por los medios de computación instrumentados a través del paradigma Turing-von Neumann, y encarnados en dispositivos electrónicos. Es probable que estos dispositivos sean sustituidos, a medio o largo plazo, por dispositivos fotónicos [consúltese D. J. Bishop et al. (2001)]. Por su parte, la concepción clásica de la computación representada por el paradigma Turing-von Neumann podría verse reemplazada, en un futuro no demasiado lejano, por el nuevo paradigma de la computación cuántica. Véanse G. Zaft (1997), A. Barenco et al. (1996), D. Deutsch (1999).

8 En este contexto, un metadato es un dato que permite el procesamiento adecuado de otros datos. Por ejemplo, la dirección IP de una computadora es un (meta)dato que permite que la máquina intercambie datos (comunicativos, efectivamente «consumibles") con otras en la Red.
} 
das, constituye ahora mismo uno de los mayores retos de la tecnología informática. Se trata de herramientas diseñadas para facilitarnos el acceso a aquellos contenidos de la Red que nos interesan; para permitirnos una selección lo más atinada posible de esos contenidos; y para ayudarnos, finalmente, a combinar y modificar éstos según nuestros objetivos y preferencias del momento.

Hacia una Internet autoorganizante, capaz de representarse y controlarse a sí misma

Para poder mejor servir las necesidades particulares de los usuarios, esas herramientas de metainformación tienen que apoyarse en representaciones dinámicas de la Red. Unas representaciones que resultan, a su vez, parcialmente generadas por algunas de esas herramientas, como los motores de búsqueda. De manera que Internet, a través de la acción de dispositivos metainformacionales específicos, tiende a producir «mapas cognitivos» de sí misma cada vez más precisos y elaborados. Por su parte, esos mapas, a medida que guían las trayectorias de navegación en la Red que ejecutan sus usuarios, contribuyen tanto a caracterizar como a reproducir la estructura de ésta de forma más y más desarrollada.

Concebir Internet como un espacio global (en los dos sentidos del término: planetario y omnicomprensivo) de información y comunicación es correcto, pero insuficiente. De manera cada vez más clara, Internet se está configurando como un sistema dotado, en varios aspectos, de la propiedad conocida como clausura organizacional ${ }^{9}$. En otras palabras: la Red lleva camino de estructurarse, progresivamente, como un sistema cuyas relaciones con su entorno - constituido esencialmente por sus usuarios - se producen y reproducen de manera relativamente estable y coherente, gracias a un conjunto de operaciones internas al propio sistema y capaces de mantener y recrear esa estabilidad y coherencia. Crece, en efecto, la evidencia de que Internet se comporta como un sistema con capacidades autoorganizativas cada vez más potentes y refinadas ${ }^{10}$. Debido a ello, ese sistema puede regenerarse y evolucionar en una dirección de mayor complejidad, pero sin perder su consistencia operativa. Como hacen también los seres vivos, Internet preserva su viabilidad como sistema a través del acoplamiento congruente entre sus operaciones externas e internas. $Y$ lo que dota a tal sistema de la coherencia que facilita ese acoplamiento es justamente la estructura metainformacional unitaria que rige su dinámica -una estructura de ámbito planetario y capaz, hasta cierto punto, de representarse y controlarse a sí misma-.

9 P. Navarro (1994).

10 Véanse F. Heylighen y J. Bollen (1996), N. Johnson et al. (1998). 
Esa estructura metainformacional es la que configura y anima el entero sistema de Internet. Por ello, es imprescindible proceder a una cuidadosa caracterización de la misma si se quiere entender tanto el funcionamiento actual de la Red como su previsible evolución futura. En la sección siguiente se esbozarán algunos conceptos aplicables al análisis de ese dominio metainformacional.

\section{DIMENSIONES Y TÉCNICAS DEL ANÁLISIS DE LA INFORMACIÓN PRESENTE EN LA RED}

La información en la Red se estructura, como ya se ha sugerido, en dos niveles fundamentales: el que representa la información de comunicación y el correspondiente a la estructura metainformacional que permite procesar la primera. Mas esta estructura, a su vez, está constituida por una pluralidad de estratos que se corresponden, por una parte, con las capas del llamado modelo $O S \Gamma^{11} \mathrm{y}$, por otra, con las distintas dimensiones implícitas en la propia información de comunicación. A este respecto, cabría distinguir dentro de esa información de comunicación entre una dimensión sintáctica, otra semántica y una tercera pragmática. Estas tres dimensiones, por lo demás, podrían también rastrearse en la estructura que el modelo OSI especifica a grandes rasgos. Veamos qué rendimiento epistémico podemos sacarle al estudio de estos tres dominios metainformacionales.

\section{a) La dimensión sintáctica}

En el plano metainformacional, la información que permite analizar los contenidos y procesos de Internet desde un punto de vista sintáctico se concreta en al menos dos niveles. El más básico de ellos se correspondería, en términos del modelo OSI, con los protocolos de red y transmisión. Es el nivel representado por el sistema TCP/IP ${ }^{12}$ y por los protocolos de transmisión de los diversos servicios (correo electrónico, web, etc.).

A continuación, en esa escala de niveles de metainformación se sitúa el estrato correspondiente al formato de los contenidos informativos. Para que esos contenidos sean perceptibles físicamente por el usuario de un modo inequívoco, claro y a la vez flexible, es preciso

11 El modelo OSI (Open Systems Interconnection) define siete capas en los procesos de comunicación entre sistemas en red (los niveles físico, de enlace, de red, de transporte, de sesión, de presentación y de aplicación). Estas siete capas representan otros tantos grados de abstracción o estructuración de la información que circula por la Red: el grado de estructuración más bajo se materializa en el nivel físico, en tanto que el más alto corresponde al nivel de aplicación. En general, cuanto más estructurada esté la información objeto de análisis, más rico será su significado.

12 Una introducción al sistema de protocolos TCP/IP puede encontrarse en S. Barajas (2001). 
darles forma de una manera estándar, universalmente aceptada. Metalenguajes de formato, como el HTML, aportan los recursos técnicos que permiten definir la apariencia física de los contenidos (el color de la página correspondiente, la presentación del texto, etc.). Los dos estratos de metainformación hasta ahora aludidos - el de los protocolos de transmisión y el del formato de los contenidos-, así como las correspondientes técnicas que los instrumentan y analizan, pueden concebirse como niveles pertenecientes a la dimensión puramente sintáctica de la estructura metainformacional que subyace al uso de la información presente en la Red.

\section{b) La dimensión semántica}

Un tercer nivel metainformacional estaría representado por la codificación semántica, según criterios explícitos, de los contenidos mismos de esa información. Es la tarea que trata de impulsar el proyecto de Web semántica acaudillado por el creador de la Web «normal», Tim Berners-Lee ${ }^{13}$. La indicada codificación se implementaría técnicamente por medio de mecanismos informáticos capaces de realizarla de forma precisa e inequívoca, de modo tal que el contenido semántico involucrado pueda ser automáticamente distinguido y procesado por cualquier computadora. Este nivel de metainformación ya no se ocuparía, pues, de la apariencia física de la información disponible en la Red, sino de los contenidos conceptuales (o significados) presentes en la misma.

Es esa clase de codificación semántica la que se pretende realizar, por ejemplo, mediante el lenguaje de anotación (o de marcación por etiquetado) XML (eXtensible Markup Langua$g^{14}{ }^{14}$, desarrollado por el World Wide Web Consortium ${ }^{15}$. Se trata de un lenguaje de anotación más poderoso, como se verá, que el HTML, al que con el tiempo parece destinado a absorber. EI XML es una adaptación para la Web de un lenguaje anterior y menos manejable, el SGML (Standard Generalized Markup Language). Más que un lenguaje de etiquetado concreto, $\mathrm{XML}$ es un esquema general que facilita la elaboración de diferentes lenguajes de etiquetado, diseñados para propósitos específicos. Esos lenguajes permiten explicitar los metadatos (datos acerca de datos) que sirven para interpretar y procesar automáticamente la información presente en cada archivo informático. Tomando como base el $\mathrm{XML}$ es posible definir no sólo el formato de los documentos disponibles en la Red, sino también sus contenidos o, si se prefiere, su semántica. Cabe así, por ejemplo, codificar los

\footnotetext{
13 Consúltense T. Berners-Lee et al. (2001), T. Berners-Lee y M. Fischetti (2000).

14 Véase $h t t p: / / w w w . w 3 . o r g / X M L /$. La especificación del XML puede encontrarse en http://www.w3.org/XML/Core/.

15 La dirección del W3 Consortium es http://www.w3.org/.
} 
conceptos particulares de los que tales documentos hablan, los autores de los mismos, su fecha de edición, las clases de elementos que incluyen, etc.

El XML se concreta a través de la elaboración de unos patrones de documento plenamente especificados, cada uno de los cuales constituye una «Definición de Tipo de Documento" (Document Type Definition, DTD). Por cierto, lo que conocemos como lenguaje HTML no es sino una DTD particular del SGML, diseñada con el objetivo de estandarizar el formato de los documentos de la Web. Una vez creada una DTD adecuada para captar la estructura metainformacional de determinada clase de documento, se trata simplemente de elaborar en lo sucesivo ese tipo de documentos según la DTD en cuestión, explicitando y etiquetando en cada caso todos los elementos especificados en ella.

A finales de los años noventa se elaboró el lenguaje RDF (Resource Description Framework $)^{16}$, que es una DTD especialmente diseñada para facilitar el procesamiento automático del contenido semántico de la Red. Este lenguaje de etiquetado está concebido para expresar diversos conjuntos de metadatos semánticos - a los que se da el nombre de ontologías ${ }^{17}$ - que serían característicos de distintas clases de documentos (por ejemplo, fichas bibliográficas ${ }^{18}$ o contratos de trabajo ${ }^{19}$ ). La explicitación de esas peculiares ontologías debe permitir el ulterior procesamiento automático de los correspondientes significados.

Así, supóngase que el documento cuyos contenidos semánticos se pretende codificar no es otra cosa que la crónica de un partido de fútbol. Con este propósito, lo que tendríamos que hacer es elaborar, a partir del lenguaje RDF y de otros instrumentos metainformacionales recientemente desarrollados, como el $\mathrm{OWL}^{20}$, la ontología «crónica de partido de fútbol». Esta ontología incluiría como elementos predefinidos, por ejemplo, el lugar y fecha del encuentro, los equipos enfrentados, sus respectivas alineaciones, el equipo arbitral, el resultado del partido, el momento en que fueron marcados los tantos y por quién, etc. A partir de la estructura de metadatos definida por esa ontología, el periodista deportivo de turno tendría que redactar su crónica rellenando con los correspondientes datos todos o parte al

\footnotetext{
16 Consúltese http://www.w3.org/TR/2004/REC-rdf-primer-20040210/.

17 Según la acepción consagrada para este término por la Inteligencia Artificial (véase http://www-ksl.stanford.edu/ kst/whatis-an-ontology.html).

18 Consúltese la ontología conocida como «The Dublin Core Metadata Initiative», en http://dublincore.org/.

19 Véase el proyecto «The Enterprise Ontology», en http://www.aiai.ed.ac.uk/project/enterprise/enterprise/ontology.html.

20 El OWL (Web Ontology Language) es un lenguaje declarativo de ontología que permite realizar inferencias (es decir, «razonar») a partir de cierta estructura de metadatos formulada por medio del lenguaje RDF. Véase http://www.w3.org/2001/ sw/WebOnt/.
} 
menos de los campos conceptuales postulados ${ }^{21}$. De este modo, es posible diseñar una estructura semántica estándar para cada tipo de documento —-pues el número de ontologías que se pueden especificar y manejar a partir de lenguajes como RDF y OWL es, en principio, ilimitado-.

El objetivo de esta forma de organizar la información no sería sólo forzar la explicitación de ésta por su autor, sino también, y como se ha señalado, posibilitar la automatización de su procesamiento semántico. Imaginemos las consecuencias que esto tiene: en todo momento y de manera casi inmediata, toda la información codificada de este modo en la Red resultaría accesible, desde cualquier punto de ésta, a través de motores de búsqueda apropiados. No sólo eso: podríamos, por ejemplo, sintetizar nuevas informaciones a partir de elementos semánticos desperdigados en multitud de documentos, y así saber, digamos, en qué minuto se marcó el gol que decidió la liga de tal o cual año en este o el otro país, o cuántas veces cierto árbitro, a lo largo de su entera ejecutoria, ha expulsado a determinado jugador.

Repárese, además, en que la utilización de un instrumento descriptivo e inferencial lo bastante potente permitiría, en principio, ampliar los contenidos de cualquier documento - digamos, la ficha de determinado jugador- gracias a la combinación de datos procedentes de diversos tipos de documentos. Esa ampliación podría hacerse sobre la marcha, de acuerdo con los requerimientos del usuario del documento en cuestión.

En realidad, la generalización de estándares de codificación semántica cada vez más potentes tendrá como resultado la conversión de la Red —o, al menos, del «universo visible» de la misma al alcance de cada usuario- en una gigantesca base de datos distribuida e hiperconectada a través de motores inferenciales. Esta base de datos estará localizada en infinidad de nodos dispersos por toda la superficie del Globo, pero mutuamente accesibles e inteligibles. Cualquier demanda compleja de información por parte de los usuarios de semejante «oráculo planetario» 22 entrañará un colosal proceso de búsqueda y síntesis informativa «inteligente». Ese proceso se llevará a cabo de manera intrincadamente concurrente a lo largo y ancho de la Red, y tendrá un carácter casi por completo automatizado — por

\footnotetext{
21 Alguien puede pensar que esta estandarización significa la imposición de un corsé inaceptable para la libertad expresiva del periodista autor de la crónica. Esto es, en parte, cierto; el uso de patrones metainformacionales siempre constriñe en cierto modo la expresividad del sujeto. Pero, al propio tiempo, su utilización potencia esa expresividad en otros aspectos. En todo caso, la aportación de los contenidos semánticos estándar no impide la presencia de otros no estandarizados, ni tiene por qué estorbar demasiado los vuelos literarios del autor.

$22 \mathrm{Ni}$ que decir tiene que la existencia de ese oráculo global suscita preguntas inquietantes: ¿quién lo controlará? O, tal vez más alarmante todavía: ¿será controlable en absoluto? En este trabajo no se intentará abordar cuestiones como éstas que, justamente por su importancia, merecen tratamiento aparte.
} 
lo tanto, en cierto modo ciego, en el sentido de invisible para la intencionalidad de cualquier agente concreto-.

\section{c) La dimensión pragmática}

Un cuarto y último nivel metainformacional de la Red es el que se corresponde con su función pragmática. El estudio de este nivel ha de centrarse en el examen de la información producida por la Red acerca del uso que de sus contenidos hacen quienes la utilizan. Con este propósito se pueden emplear diversas técnicas. En primer lugar, cabe recurrir al análisis de las vinculaciones entre los nodos de la Red - tanto nodos servidores como nodos clientes-. Este análisis, en cierta forma, nos revela la «personalidad de red» de los usuarios habituales de tales nodos. Se trataría, en este sentido, de caracterizar los nodos de la Red según los contenidos que albergan, emiten o demandan, y según con qué otros nodos se conectan como emisores o receptores de información. Mediante el establecimiento de estos perfiles de uso dispondríamos de una primera visión, por rudimentaria que fuera, de la pragmática de la Red.

\section{- Filtrado colaborativo}

La caracterización pragmática de la Red puede beneficiarse de otras técnicas de análisis. Así, es posible escudriñar las vinculaciones no ya entre los nodos, sino entre los documentos de la Red. No se trataría, en este caso, de rastrear sus contenidos semánticos comunes, sino de considerar otros aspectos propiamente pragmáticos, como cuáles son los documentos explícitamente referidos por cada documento y cuáles son los que explícitamente se refieren a éste. Por ejemplo, si el documento es una página personal, resulta interesante saber a qué otras páginas se vincula tal documento —algunas de ellas, presumiblemente, páginas personales a su vez-y qué otros documentos —quizá también páginas personales - se vinculan al mismo. Mediante este análisis ${ }^{23}$ sería posible establecer interesantes vínculos sociométricos entre documentos y, en definitiva, entre usuarios de la Red.

Los documentos pueden vincularse con otros documentos no sólo directamente, sino también atendiendo a los usuarios comunes que comparten. Parece lógico pensar que documentos con frecuencia demandados por los mismos usuarios deben tener algún contenido

\footnotetext{
${ }^{23}$ Una versión de este método analítico, denominada PageRank, es usada por el buscador Google. Véase http://www.google. es/intl/es/why_use.html.
} 
semántico, pero sobre todo pragmático, común. Este planteamiento es el que utilizan algunas técnicas de Collaborative Filtering o Social Filtering ${ }^{24}$ desarrolladas en el entorno de Internet y que han sido popularizadas por sitios tan influyentes como Amazon.com ${ }^{25}$. Esta librería en la Web elabora listas de recomendaciones que ofrece a sus clientes. Según parece, esas listas se componen a partir de algún tipo de análisis de correspondencias. Ésta es una técnica estadística mediante la que, en este caso, se seleccionan para cada cliente ciertos libros: precisamente aquellos que han sido comprados por otros clientes que también han adquirido algunos de los libros ya demandados por el cliente en cuestión ${ }^{26}$. La idea que da sentido a este procedimiento es simple, pero elegante. Se supone que aquellos clientes que han comprado los mismos libros deben tener gustos parecidos; y, por tanto, las nuevas adquisiciones de algunos de esos clientes pueden resultar también interesantes para los demás.

Lo más fascinante de este mecanismo es que, a través de él, son las decisiones de otra gente perfectamente anónima e indeterminada para ego las que, en definitiva, suministran a éste la información que probablemente vaya a determinar sus propias decisiones. Nos encontramos así con una suerte de interacción social indirecta entre unos agentes que, como tales, se mantienen en una condición de opacidad mutua casi perfecta. Unos agentes que, con todo, encuentran la forma de sincronizar recíprocamente sus acciones - al menos, en un sentido probabilístico - como guiados por la mano invisible que encarna un algoritmo estadístico ejecutado de forma enteramente transparente (es decir, imperceptible). Volveremos más adelante sobre el significado de este tipo de interacción. Mas conviene ya subrayar que estas vinculaciones indirectas e implícitas entre personas pueden engendrar interacciones sociales bien reales, pero difíciles de captar con el instrumental analítico tradicional.

De la misma forma que los documentos pueden relacionarse con otros documentos a través de los usuarios que los comparten, los usuarios pueden relacionarse con otros usuarios por medio de los documentos que acceden conjuntamente. En efecto, los usuarios no sólo definen su «personalidad social» en Internet a través de su relación con los otros usuarios con los que se conectan directamente. Lo hacen también por medio de las vinculaciones indirectas que mantienen con aquellos otros usuarios, probablemente desconocidos, que comparten con ellos el acceso a determinados recursos de la Red.

\footnotetext{
24 Consúltese http://pespmc1.vub.ac.be/COLLFILT.html. El concepto de Collaborative Filtering es en buena medida isomorfo con el de análisis sociosemántico, propuesto en la primera mitad de los noventa, y con vistas a su aplicación en la investigación sociológica, por C. Díaz Martínez y yo mismo [véanse P. Navarro y C. Díaz Martínez (1994), C. Díaz Martínez (1996)].

25 Localizable en http://www.amazon.com.

${ }^{26}$ El análisis que se comenta es en realidad algo más complejo, pues acepta asimismo que el cliente exprese directamente sus preferencias.
} 


\section{- Agentes informáticos}

Una utilización eficaz de la plétora de información que Internet ofrece al usuario requiere algo más que mecanismos metainformacionales capaces de producir «mapas cognitivos» detallados y actualizados de Internet. Pues esos «mapas macro» seguirán siendo difíciles de manejar por los usuarios de la Red mientras ésta no disponga de los «mapas micro» correspondientes a cada uno de esos usuarios a los que, en definitiva, tiene que servir. No basta simplemente con que Internet ofrezca sus contenidos a un usuario genérico. La Red debe también «conocer» a la persona concreta que la usa, pues debe adelantarse proactivamente a los requerimientos de ésta con el objeto de minimizar los costes de su búsqueda de información personalmente relevante.

Lo que sería útil, en este sentido, es que fuera la Red, en lugar de cada usuario, la que asumiera la tarea de seleccionar para ese usuario singular los contenidos y las posibilidades de comunicación que, previsiblemente, van a interesarle. Mas ello requiere que la Red disponga de información lo más relevante posible acerca del usuario en cuestión. No información de tipo sociodemográfico ${ }^{27}$, sino mucho más concreta: información de carácter auténticamente personal. Por ejemplo, cuáles son los libros que yo he comprado en los últimos meses, si determinada persona que me envía un corr.el ${ }^{28}$ es un desconocido o alguien con quien mantengo correspondencia habitual, cuáles son las páginas web más visitadas por mí, si hay otras páginas de contenido similar que me han pasado desapercibidas...

Para cubrir esa demanda potencial, en los últimos tiempos se vienen desarrollando programas destinados a elaborar «mapas cognitivos» —o, más bien, «mapas cognitivo-pragmáticos»- de los usuarios individuales de Internet. Cuando esos programas lleguen a aplicarse, la trayectoria de navegación de cada internauta se convertirá en un proceso guiado por el acoplamiento dinámico entre dos estructuras metainformacionales. Por un lado, los mapas de la Red global —y de cualquiera de sus subconjuntos-. Por otro, el perfil semánticopragmático de ese usuario concreto. Este perfil se construiría a partir de cierta metainformación idiosincrásica, dinámica y permanentemente actualizada acerca de sus intereses, hábitos y singladuras como internauta.

En general, los programas concebidos para remedar el tipo de actividades que desarrollan los usuarios de la Red en sus tratos con ésta reciben el nombre de software agents ${ }^{29}$. Los

\footnotetext{
27 Véase N. Negroponte (2000), pp. 241-252.

28 En francés se ha propuesto la contracción courri.el como traducción del inglés e-mail. ¿Por qué no utilizar en español el equivalente corr.el (plural, corr.eles)? Creo que es un término a la vez fonológicamente aceptable y semánticamente expresivo.

29 Véanse J. Hendler (2001), J. Sverker (2002), CoABS (2004).
} 
más elaborados de estos agentes informáticos merecen a menudo la denominación de autonomous software agents, porque pueden realizar su tarea con un mínimo control externo. Además, muestran cierta capacidad de aprendizaje, que les permite adaptarse a su peculiar entorno.

Pues bien, de entre tales agentes, aquellos concebidos para recabar y registrar automáticamente la metainformación acerca del usuario a la que estamos aludiendo, y para utilizarla dinámicamente de la manera sugerida, reciben el nombre de «agentes informáticos personales» (en inglés, personal software agents). Estos agentes son programas diseñados para definir un determinado perfil —o más bien un «rastro», pues se trata de algo a la vez concreto y dinámico- de usuario individual, y para satisfacer los requerimientos informativos especificados por ese rastro. Con este objetivo, los agentes en cuestión realizan sus operaciones a partir, por una parte, de la metainformación específica que generan acerca del usuario al que sirven y, por otra, de toda la rica estructura metainformacional de la Red.

Por ejemplo, el MediaLab del Instituto de Tecnología de Massachusetts viene desarrollando agentes informáticos ${ }^{30}$ capaces de realizar operaciones cada vez más elaboradas. Así, el programa Letizia «asiste al usuario que navega la Web, aprendiendo sus intereses y explorando más allá de la posición actual de ese usuario, para encontrar páginas de posible interés»; Amalthea «es un ecosistema artificial de agentes evolutivos de descubrimiento y filtrado de información, que cooperan y compiten en un entorno similar al de un mercado»; Footprints «es una aplicación del concepto de historia de interacción al problema de la navegación social; esto es, el uso de información dejada por otra gente para ayudarle a definir su trayectoria».

\section{4. ¿UNA NUEVA DIMENSIÓN DE LA SOCIALIDAD HUMANA?}

Las repercusiones sociales que va a tener la emergencia de todo este dominio metainformacional encarnado por Internet son profundas. Mas para sondearlas es preciso tomar distancia ante el complejo fenómeno que nos ocupa y visualizarlo desde una perspectiva más amplia —en su dimensión genuinamente sociohistórica-.

\section{Internet como sistema de interacción social}

Desde un punto de vista propiamente sociológico, Internet no es simplemente un medio $-\mathrm{y}$, según hemos visto, un genuino factor- de comunicación. Es eso, pero también algo

\footnotetext{
30 Consúltese MIT MediaLab (2004).
} 
más: un complejo sistema de acción e interacción social. Éste es el hecho que convierte la elaborada estructura metainformacional que da vida a la Red en una realidad de interés no meramente técnico y especializado - restringido, mayormente, a los expertos en nuevos medios de comunicación-, sino más general. Porque esa estructura, más allá de su carácter comunicacional, resulta ser, por sus dimensiones y efectos, un auténtico sistema de interacción de dimensiones planetarias -y por ello una estructura social en el pleno sentido de la expresión-. De ahí su relevancia ineludible para la teoría y la práctica sociológicas.

Internet es un caso claro de estructura social emergente, expresión y vehículo de una nueva dimensión de la socialidad humana. Esta novedosa dimensión se está articulando más rápidamente de lo que imaginamos. Y lo hace impulsada por la aparición de una forma inédita de vínculo social: el generado por cierto modo peculiar de interacción, guiada por una intencionalidad transconsciente ${ }^{31}$, artefactual y objetiva, que se halla encarnada en la maquinaria metainformacional de la Red. El desarrollo de ese nuevo tipo de vínculo social va a entrañar, con toda probabilidad, la constitución de una sociedad bien distinta de las anteriores, dotada de nuevos niveles de complejidad y configurada a la medida de su talla genuinamente global.

\section{La peculiaridad de la interacción social en Internet}

Los vínculos sociales que somos capaces de establecer por medio de la Red —a través de los distintos entornos de interacción a los que ella nos brinda acceso- son muy diversos. Podemos, por ejemplo, practicar ese simulacro casi perfecto de interacción cara a cara que es la videoconferencia. Podemos «chatear» por medio del teclado, o intercambiar documentos de todo tipo gracias al correo electrónico. O podemos tal vez preferir usar Internet para escuchar un medio «clásico» como la radio... Por otra parte, la facilidad con que en el seno de Internet surgen "comunidades virtuales» 32 parece dar un sentido inopinado a la vieja noción de «aldea planetaria» propuesta por McLuhan ${ }^{33}$. Esta aldea, ahora, ya no se nos mostraría como una macrocomunidad mundial unificada por los medios de masas, sino más bien como una infinidad de pequeñas colectividades, todas ellas distribuidas y activas de manera multiplexada a lo largo y ancho de la Red.

\footnotetext{
31 Es decir, una intencionalidad que, como se explicará a continuación, se constituye en un espacio sui generis, generado por una suerte de entrecruzamiento «objetivo» de las conciencias individuales.

32 Véase H. Rheingold (1996).

33 M. McLuhan (1996).
} 
Miradas las cosas más de cerca, sin embargo, fácil es advertir que todos esos entornos de interacción no son las realidades más peculiares y novedosas que la Red nos ofrece. Pues interacciones como las que permite la videoconferencia, o el correo electrónico, no son sino remedos, o versiones tecnológicamente potenciadas, de formas interactivas previamente existentes, como la interacción natural cara a cara, el teléfono o el correo tradicional. Sin duda, la posibilidad de interactuar, directamente y en tiempo real ${ }^{34}$, con personas situadas a miles de kilómetros de distancia entraña una variación radical en la topología del espacio social. La puesta en práctica de esa posibilidad por parte de buen número de ciudadanos va a modificar drásticamente su percepción del mundo en general, y de la realidad social en particular — un hecho que, por sí solo, tendría ya consecuencias históricas de gran alcance-.

Mas de entre todos los entornos de interacción posibilitados por la Red, aquellos que van a tener probablemente mayor peso en la transformación de nuestras sociedades $-\mathrm{y}$ en la rápida eclosión de una sociedad genuinamente planetaria- no son éstos que se acaban de mencionar, sino otros. En lugar de ser los entornos que facilitan interacciones personales directas, serán más bien los que permiten interacciones indirectas, anónimas y ciegas entre innumerables agentes mutuamente desconocidos e inaccesibles. Estas interacciones no se organizan por medio de la intencionalidad individual de las conciencias interactuantes, sino gracias a una suerte de intencionalidad transconsciente encarnada en el propio sistema global de información que es la Red. Esta intencionalidad tiene un carácter artefactual y objetivo; está representada por la entera maquinaria metainformacional de la Red y, de alguna manera, "utiliza» las conciencias de los agentes individuales como la fuente de energía e información que le permite reproducirse y evolucionar. Los entornos de interacción transconsciente a los que me refiero, a diferencia de los que se fundan en las capacidades mutuamente reflectivas de las conciencias individuales ${ }^{35}$, tienen un carácter casi totalmente opaco y se definen, como veremos, de modo virtual antes que real ${ }^{36}$. Tales entornos están instrumentados a través de mecanismos que, por todos esos rasgos, recuerdan el tipo de funcionamiento propio del mercado, en su papel de instrumento globalmente ciego —en realidad, transconsciente— mediador de las interacciones económicas.

\footnotetext{
34 A un coste ampliamente asequible, además. La videoconferencia —vía satélite- y no digamos el teléfono a larga distancia han sido realidades tecnológicas disponibles durante decenios. Pero el coste de una y otro era tan elevado que hasta la popularización de Internet esas formas de interacción no han tenido posibilidad alguna de generalizarse.

$35 \mathrm{La}$ intencionalidad propiamente humana, en la medida en que se articula por medio de una conciencia capaz de reflejar otras conciencias, tiene un carácter no sólo congénitamente transconsciente, sino transintencional. Sin embargo, esa transintencionalidad es de naturaleza subjetiva: depende, justamente, de esa condición de reflexión entre las conciencias que relaciona. La transintencionalidad a la que aquí se alude, por el contrario, es de naturaleza «objetiva»: no depende de la indicada reflectividad, sino de la concurrencia de facto entre conciencias relacionadas sólo externamente, a través del aparato metainformacional que la Red pone a su disposición. Véase P. Navarro (1994).
}

36 Aquí «real» se emplea en el sentido de actual, como se puntualizará más adelante. 


\section{La Red como dispositivo de interacción virtual}

El desarrollo de la interacción humana mediada e indirecta ha podido producirse gracias a la aparición, a lo largo de la historia, de dispositivos de interacción virtual —como los he llamado en algún otro lugar ${ }^{37}$ — cada vez más poderosos. Un dispositivo de interacción virtual (verbigracia, la escritura o el dinero) es un mediador ${ }^{38}$ de interacciones potenciales indirectas. Estas interacciones, al realizarse con éxito a través de ese dispositivo, ponen en relación, y sincronizan de algún modo, las conductas de los agentes por él vinculados.

El carácter "virtual»39 de la interacción que esos dispositivos instrumentan viene dado por la peculiar distancia que establecen entre los agentes que, en principio, pueden interactuar a través de ellos. Pongamos un ejemplo: un texto escrito, en sí mismo, no articula una interacción real. Sólo lo hace cuando es efectivamente leído - y entendido- por alguien distinto de su autor. Ese alguien a menudo es una persona a la que este autor no conoce. De ser así, se trata de una persona inexistente como realidad concreta -0 , si se quiere, existente sólo como realidad virtual- en la mente de tal autor mientras escribe el texto en cuestión. Obsérvese, pues, que un texto, a pesar de no constituir en sí mismo una interacción real (en el sentido de actual), es ya, en el propósito de quien lo escribe, una interacción incoada - virtual, y en este sentido real, aunque no enteramente realizada (no actualizada) todavía- Cabría postular que una interacción virtual es una suerte de función interactiva —noción similar a la de «función proposicional» en lógica-. Una función proposicional es un esquema enunciativo abierto que, cuando ve reemplazadas sus variables por valores concretos (llamados argumentos), se convierte en una proposición. De manera similar, una función interactiva sería un esquema de interacción en principio abierto, pero que puede concretarse tomando como argumentos diferentes valores — por ejemplo, todos y cada uno de los posibles lectores capaces de satisfacer la función «leer el texto a del autor $b »-$.

Desde el punto de vista que aquí se postula, la Red representa la aparición de un dispositivo de interacción virtual de nuevo tipo, al menos tan complejo y potente como cualquiera de los que lo han precedido. Es un instrumento que permite y vehicula, además de un amplio abanico de interacciones directas entre agentes —en el plano que llamaremos inter-

\footnotetext{
37 Véase P. Navarro (1999).

38 Un mediador, se entiende, de naturaleza artificial. Los seres humanos también somos «mediadores de interacciones indirectas» entre otros seres humanos; pero somos mediadores naturales, no artefactos diseñados con ese propósito.

39 Por virtual entiendo aquello que, dentro de cierto sistema, tiene una realidad sólo potencial, pero puede convertirse en actual de acuerdo con la dinámica propia de ese sistema. Dicho de otro modo, lo virtual es una suerte próxima de potencialidad: es lo potencial en tanto que congruentemente actualizable dentro de cierto sistema (o, lo que es lo mismo, de cierta configuración de la realidad).
} 
personal-, interacciones indirectas, virtuales y, sin embargo, extraordinariamente poderosas en el plano que podríamos denominar transpersona ${ }^{40}$.

\section{¿Una sociedad capaz de autocomputarse a sí misma?}

Así pues, en la Red la interacción social no tiene por qué producirse sólo en un formato interpersonal: puede autocomputarse a sí misma a partir de las acciones individuales de sujetos indirectamente interactuantes. Estas acciones proporcionarían los datos cuya elaboración metainformacional -a través de programas adecuados, como los agentes informáticos - tendría la virtud de generar la información —o, si se prefiere, el conocimiento- que, a su vez, contribuiría a decidir el curso y el contenido de la acción de esos mismos sujetos. Todo ello ocurriría en tiempo real - a medida que esa acción se va desarrollandoy sin que en el indicado proceso de elaboración metainformacional tengan que intervenir ni la voluntad ni la conciencia de los sujetos involucrados. Éstos, al ejecutar sus trayectorias individuales como agentes, y aunque no sean capaces de percibirse los unos a los otros, no pueden sino influirse mutuamente a través de la representación estadística que del conjunto de sus trayectorias les ofrecen los dispositivos informáticos de interacción virtual mencionados. Poca duda cabe, por tanto, de que tales sujetos, en el acto mismo de determinar su acción por medio de esa representación, están sincronizando de algún modo sus conductas y, por ende, se encuentran interactuando de una forma implícita pero eficaz.

Esta interacción social virtual, de tipo transpersonal y reificado, que la Red acoge y fomenta, es en más de un aspecto similar — para seguir con la analogía previamente apuntada-

\footnotetext{
40 Habría que tener en cuenta un tercer plano, el subpersonal. A lo largo de este trabajo se ha podido dar la impresión de que quienes interaccionan con Internet son siempre personas. No es así. También pueden interaccionar con la Red cosas —que interaccionarán en un sentido distinto del humano, por supuesto-. De hecho, a lo largo de los próximos años no sólo vamos a asistir a una consolidación del uso de Internet por parte de la gente, y a un progresivo afianzamiento de la Red en prácticamente todos los dominios de la vida social. Vamos a presenciar asimismo una auténtica capilarización de la presencia de Internet en el interior de nuestra propia cultura material. Las cosas mismas -incluso las más vulgares y corrientes- van a empezar a estar en red —conectadas a ésta, como suministradoras constantes de información acerca de sí mismas y de las relaciones que mantienen con su entorno- . O, dicho de otro modo: Internet está abocada a desarrollar sensores propios, tan numerosos y omnipresentes que en unos pocos decenios van a recubrir el entero espacio de nuestra cultura material y de nuestro ecosistema - transformando la Red en una especie de duplicado informacional de ese espacio-. Internet se convertirá así en una suerte de Mapa Imperial borgiano — coextensivo con el territorio que representa— en versión digital. No sólo los ordenadores: los coches, los ascensores, los niveles de los ríos, los incendios forestales, van a estar en red. Incluso los cartones de leche y los paquetes de azúcar de los supermercados estarán previsiblemente «conectados» mediante etiquetas activas e inteligentes, que informarán a la Red, por ejemplo, de que han sido comprados en tal sitio, a cierta hora, a determinado precio, pagado mediante una tarjeta con tal número por esta o la otra persona. Llamo «interacción subpersonal» a esta clase de interacción directa de la Red con el ecosistema social, a través de las cosas mismas y sin la intervención directa de personas. Cuando se tiene en cuenta la actividad de Internet también en este nivel, ésta se nos revela como algo más que un medio y un factor de comunicación: se nos muestra como medio y factor directo de organización de la vida social. Esta nota resultará hoy en día obvia para cualquiera que conozca desarrollos recientes como el de las etiquetas EPC (Código Electrónico de Producto), basadas en la tecnología RFID (IDentificación por RadioFrecuencia) y que parecen destinadas a sustituir los actuales códigos de barras; véanse R. Want (2004) y la revista electrónica RFID Journal [http://www.rfidjournal.com].
} 
a la que vincula a la mayoría de los agentes económicos actuantes en un mercado. En ambos casos se trata de una interacción social «a distancia», anónima y opaca, que se produce sin que por fuerza exista una conciencia recíprocamente reflectiva entre los agentes involucrados en la misma - pero que sin embargo genera, según se ha intentado mostrar, cierta forma de reciprocidad-. Como ocurre con las relaciones de intercambio mediadas por dinero, la interacción virtual en la Red se convierte en un mecanismo de constitución societal ultraconsciente, en el sentido de que se halla situado más allá del control de cualquier conciencia individual. Empero, son las conciencias individuales las que, actuando siempre en un plano local y a través de sus propias operaciones cognitivas y agenciales, suministran al sistema la información básica que éste luego sintetiza y devuelve, debidamente transformada, a los individuos.

Los mecanismos que posibilitan la interacción virtual de los individuos en Internet encarnan, pues, una suerte de intencionalidad objetiva incorporada en su propio diseño ${ }^{41}$, y subsumen en un dominio transintencional las diversas actividades intencionales de los agentes por ellos vinculados. Así, esos mecanismos engendran resultados que se sitúan fuera del control de cualquier agente -y ello a pesar de que tales resultados no son sino el efecto de la concurrencia, ponderada por medio de procesos de cómputo más o menos complejos, de las intenciones individuales de todos esos agentes-.

\section{Recapitulación y perspectivas}

En la Red, como hemos visto, la clase de interacción social virtual que se ha denominado transpersonal puede autocomputarse en el propio proceso de su despliegue, en tiempo real y de forma automática. La trayectoria misma de la acción del usuario de la Red resulta de este modo definida, en parte, por esa interacción autocomputada (es decir, por esa interacción que resulta computada en y por el propio proceso en el que la misma se despliega). De este modo, el curso que sigue la navegación de cada usuario por Internet se convierte en una secuencia de interacciones sociales implícitas, pertenecientes a un espacio interactivo de nuevo tipo, producido y reproducido por la misma experiencia de uso de la Red.

No parece demasiado aventurado suponer que, a partir de esta novedosa forma de interacción virtual, va a producirse la emergencia de esa nueva dimensión de la socialidad

\footnotetext{
41 Los instrumentos, en general (pensemos en un martillo o en un cepillo de dientes), son constructos intencionales objetificados. Los mecanismos de interacción virtual en red son, en este sentido, instrumentos que objetifican el propósito agencial para el que han sido diseñados. Un propósito que sólo puede cumplirse a través de las elaboradas interacciones que esos mecanismos vehiculan.
} 
humana de la que ya se ha hablado: la socialidad autocomputada o, si se prefiere, la socialidad artificial ${ }^{42}$. Esta dimensión inédita del hecho social humano que ahora comienza su despliegue está constituida por lazos apenas visibles todavía, pero que ya comienzan a hacer sentir su gentle force ${ }^{43}$ en su calidad de urdimbre de un nuevo ámbito sociogenético coextensivo con la propia Red.

\section{EL NUEVO PAPEL DE LOS MÉTODOS DE INVESTIGACIÓN SOCIAL EN LA ERA DE LA SOCIALIDAD ARTIFICIAL}

¿Cuáles van a ser las consecuencias de todas estas transformaciones para las técnicas de investigación social? De entrada, parece claro que se va a producir un cambio sustancial en el objeto mismo de esa investigación. La sociedad informacional, articulada mediante procesos de autocomputación en red de sus interacciones, adquirirá sin duda perfiles muy distintos de los que fueron típicos de la sociedad documental y mediática que la ha precedido. Es esa mutación histórica de largo alcance la que va a imponer nuevas exigencias y tareas a las técnicas del análisis sociológico.

Antes de la aparición de la Red, el conocimiento sociológico debía ser conscientemente producido y conscientemente asimilado por los actores sociales para que pudiera ejercer su característico efecto sociogenéticamente reflexivo (es decir, para que volviera, en forma de prácticas sociales, a su objeto teórico originario, la sociedad). En la Red, por el contrario, ese conocimiento se produce y difunde, en gran parte, de manera no consciente - como consecuencia, según hemos visto, de la actividad de un buen número de instrumentos metainformacionales-. Es un conocimiento, además, que resulta asimilado por los usuarios de la Red de forma igualmente inadvertida, al presentarse embebido en el curso mismo de la acción de éstos.

En el nuevo entorno sociotécnico encarnado por Internet, las singularidades reflexivas típicas de anteriores fases de la modernidad —representadas por los científicos sociales y los

42 En un sentido lato, cabe entender por «socialidad artificial» toda aquella forma y capacidad de relación social que utiliza como medio algún instrumento distinto de la interacción cara a cara, congénita para nuestra especie. La escritura, por ejemplo, sería uno de esos instrumentos artificiales de relación social —unos instrumentos a algunos de los cuales se ha dado antes el nombre técnico de «dispositivos de interacción virtual»-. En consecuencia, si la noción se concibiese con un significado tan amplio, el desarrollo de la «socialidad artificial» habría comenzado casi en los albores de la civilización. Como hemos visto, sin embargo, la Red - por efecto sobre todo de las capacidades que tiene de autocomputar sus propios procesos- es algo más que un medio de interacción. Es, más bien, una auténtica fábrica de interacciones. Y este hecho le procura una potencia incomparable como instrumento de interacción artificial. No parece por ello inapropiado reservar el término «socialidad artificial» —entendido en su acepción más restringida y específica- para apelar al peculiar modo de interacción transpersonal que Internet hace posible.

43 Para usar la expresión con que Hume caracteriza la asociación de ideas. Véase D. Hume (1978), p. 10. 
líderes de opinión divulgadores de las elaboraciones de los primeros- estallan en una proliferación casi infinita. Hasta la irrupción de la Red, científicos sociales y líderes de opinión ponían a la sociedad en relación reflexiva con el conocimiento sociológico a través de unos (relativamente) pocos puntos de contacto. En nuestros días, esos puntos de contacto se multiplican, pues se producen en el seno de las (inter)acciones cotidianas de los usuarios de la Red. Así, la referida relación reflexiva se (re)produce ahora, a través de Internet, de manera tan densa como subrepticia; en el curso mismo de las acciones más corrientes de sus usuarios y de manera transparente — sin que éstos se den apenas cuenta de ello-.

En esas circunstancias, el nivel reificado de la realidad social, cuyo conocimiento era responsabilidad y patrimonio privilegiado del economista y del sociólogo, ya no adquiere una representación elaborada sólo a través de éstos. Se hace también presente en la vida social —no teórica, sino pragmáticamente ${ }^{44}$ y de forma en general inconsciente- por medio de los dispositivos metainformacionales que, cada vez más, están llamados a informar las acciones de los usuarios de la Red. Tales dispositivos, si bien han sido diseñados, en definitiva, por expertos humanos — posiblemente sociólogos_- actúan de manera crecientemente autónoma y, como resultado de su actividad, generan una suerte de conocimiento social implícito que reside de manera densamente distribuida en el entero ecosistema de la Red. Ese «conocimiento implícito» no es ya, a diferencia del viejo conocimiento del experto (sociólogo, economista...), el trasunto teórico de una realidad reificada emergente. Más bien al contrario: tal conocimiento implícito equivaldría a la conversión práctica del conocimiento consciente de experto en reificación emergente.

Así, en el contexto de socialidad artificial que Internet encarna e instrumenta, las técnicas de investigación social deben cambiar sustancialmente de carácter. Y ello porque, como ya se ha sugerido, la mayoría de esas técnicas — desde el análisis de contenido a las diversas formas de análisis multivariante - parecen estar abocadas a sufrir una profunda transformación en su modo de uso en el nuevo entorno representado por la Red. De ser instrumentos al servicio de un conocimiento especializado de tipo teórico, elaborado por profesionales de las ciencias sociales, esas técnicas van a transformarse en mediadoras directas, en tiempo real, de las interacciones sociales en la Red.

\footnotetext{
44 No todo conocimiento pragmático requiere una representación consciente, ni mucho menos una representación de tipo teórico. Por ejemplo, sabemos (en la práctica) ir en bicicleta, pero por lo general no sabemos (ni teórica ni conscientemente) cómo lo hacemos.
} 


\section{BIBLIOGRAFÍA}

AUSTIN, J. L. (1984): Cómo hacer cosas con palabras, Barcelona, Paidós.

BARAJAS, S. (2001): «Curso de protocolos TCP/IP», en http://www.saulo.net/pub/tcpip/index.html\#1

BARENCO, A., et al. (1996): «A short introduction to quantum computation», en http://www.qubit.org/library/ intros/comp/comp.html

BERNERS-LEE, T., y FISCHETTI, M. (2000): Tejiendo la Red, Madrid, Siglo XXI.

BERNERS-LEE, T., et al. (2001): «La red semántica», Investigación y Ciencia, 298, julio, pp. 38-47.

BISHOP, D. J., et al. (2001): «The rise of optical switching», Scientific American, 284, enero, pp. 88-94.

CASTELLS, M. (2001): La era de la información. I. La sociedad red, Madrid, Alianza Editorial.

CoABS (2004): «Control of Agent-Based Systems», en http://coabs.globalinfotek.com/

DEUTSCH, D. (1999): La estructura de la realidad, Barcelona, Anagrama.

DÍAZ MARTíNEZ, C. (1996): El presente de su futuro. Modelos de autopercepción y vida entre los adolescentes españoles, Madrid, Siglo XXI.

FERRATER MORA, J. (1979): Diccionario de filosofía, Madrid, Alianza Editorial, vol. 3, pp. 2211-13.

HENDLER, J. (2001): «Agents and the Semantic Web», en http://www.cs.umd.edu/users/hendler/AgentWeb.htm/

HUME, D. (1978): A Treatise of Human Nature, Oxford, Clarendon Press.

HEYLIGHEN, F., y BOLLEN, J. (1996): «The World-Wide Web as a Super-Brain: from Metaphor to Model», en http:// pespmc1.vub.ac.be/papers/WWWSuperBRAIN.html

JOHNSON, N., et al. (1998): «Symbiotic Intelligence: Self-Organizing Knowledge on Distributed Networks, Driven by Human Interaction», en Sixth International Conference on Artificial Life, Los Angeles, Universidad de California, 26-29 de junio (disponible en http://www.cs.ucsd.edu/ rik/alife6/papers/SY51.htmI).

LAMO DE ESPINOSA, E. (1990): La sociedad reflexiva, Madrid, CIS.

McLUHAN, M. (1996): Comprender los medios de comunicación: las extensiones del ser humano, Barcelona, Paidós.

MIT MediaLab (2004): «Software Agents Group», en http://agents.media.mit.edu/

NAVARRO, P. (1994): El holograma social. Una ontología de la socialidad humana, Madrid, Siglo XXI.

- (1999): «Las dos formas de la reflexividad social humana: reflexividad reflectiva y reflexividad disipativa», en R. Ramos Torre y F. García Selgas (eds.), Globalización, riesgo, reflexividad. Tres temas de la teoria social contemporánea, Madrid, CIS, pp. 333-371.

- (2002): «Información, comunicación, conocimiento y agencia en la era de la socialidad artificial», en J. M. García Blanco y P. Navarro (eds.), ¿Más allá de la modernidad? Las dimensiones de la información, la comunicación y sus nuevas tecnologías, Madrid, CIS, pp. 105-133.

NAVARRO, P., y DíAZ MARTíNEZ, C. (1994): «Análisis de Contenido», en J. M. Delgado y J. Gutiérrez (eds.), Métodos y técnicas cualitativas de investigación en ciencias sociales, Madrid, Síntesis, pp. 177-224.

NEGROPONTE, N. (2000): El mundo digital, Madrid, Suma de Letras (Biblioteca de Bolsillo). 
RHEINGOLD, H. (1996): La comunidad virtual: una sociedad sin fronteras, Barcelona, Gedisa.

SVERKER, J. (2004): «Agent-Based Systems», en http://www.agentbase.com/survey-main.htm/

WANT, R. (2004): «Sistemas de identificación por radiofrecuencia», Investigación y Ciencia, 330, marzo, pp. 40-49.

ZAFT, G. (1997): “Von Neumann Architecture as a Kuhnian Paradigm», en http://www.zaft.org/gordon/engr696a/ paper.htm

Social research techniques - from content analysis to the assorted forms of multivariate analysis - are set to undergo a fundamental transformation as a result of the emergence of the Internet phenomenon. Those techniques are going to turn from being tools that serve specialized knowledge into direct mediators, in real time, of social interactions on the Net. Over the Internet, some forms of social interaction are able to self-compute their own incidences as they develop; thanks to the Net, it is possible to perform this self-computation in real time and in an automatic way. And so it is that when implemented on the Internet, many of the classic techniques of social research become techniques of virtual interaction, giving rise to a new dimension of human sociality. An artificial sociality whose development is likely to have consequences as momentous as those entailed by other devices of virtual interaction formerly invented - for instance, money.

Key words: Sociology of the Internet, Sociological Theory, Social Research Techniques, Artificial Sociality, Social Self-computing, Virtual Interaction, Metainformation. 